Sharif University of Technology
Scientia Iranica
SCIENTIA $\quad \begin{gathered}\text { Transactions D: Computer Science Es Engineering and Electrical Engineering } \\ \text { I RAN I C A }\end{gathered}$

\title{
Stability of nonlinear uncertain Lipschitz systems over the digital noiseless channel
}

\author{
A. Farhadi* \\ Department of Electrical Engineering, Sharif University of Technology, Tehran, Iran.
}

Received 31 October 2015; received in revised form 25 September 2016; accepted 16 January 2017

\author{
KEYWORDS \\ Networked control \\ system; \\ Lipschitz nonlinear \\ system; \\ Uncertain dynamic \\ system; \\ The digital noise-less \\ channel.
}

\begin{abstract}
This paper is concerned with the stability of nonlinear Lipschitz systems subject to bounded process and measurement noises when transmission from sensor to controller is subject to distortion due to quantization. A stabilizing technique and a sufficient condition relating transmission rate to Lipschitz coefficients are presented for almost sure asymptotic bounded stability of nonlinear uncertain Lipschitz systems. In the absence of process and measurement noises, it is shown that the proposed stabilizing technique results in almost sure asymptotic stability. Computer simulations illustrate the satisfactory performance of the proposed technique for almost sure asymptotic bounded stability and asymptotic stability.
\end{abstract}

(C) 2018 Sharif University of Technology. All rights reserved.

\section{Introduction}

\subsection{Motivation and background}

Recently, stabilizing a dynamic system over a communication channel subject to imperfections (e.g., quantization distortion) has become an important problem. Some examples of systems that require to be stabilized over communication channels subject to imperfections are smart drilling system using borehole telemetry via drilling string $[1,2]$ and distributed monitoring system of oil fields. In these systems, transmission between sub-components (e.g., sensor, controller, actuator) is subject to imperfections, such as quantization distortion. Some results addressing basic problems in state estimation and/or stability of dynamic systems over communication channels subject to imperfections can be found in [3-17]. In [15], the authors addressed the problem of state estimation of an uncontrolled noiseless nonlinear Lipschitz system over the digital noiseless

\footnotetext{
* Fax: +98216602326

E-mail address: afarhadi@sharif.edu
}

channel with asymptotically zero mean square estimation error. Farhadi and Ahmed [16] addressed the problem of state estimation of distributed uncontrolled Lipschitz systems subject to bounded process and measurement noises over the packet erasure network with bounded mean absolute estimation error and Nair et al. [12] addressed the stability problem of nonlinear noiseless systems over the digital noiseless channel.

This paper is concerned with a basic problem in the stability of nonlinear dynamic systems subject to uncertain transmission as described in Figure 1. The block diagram of Figure 1 has been considered in several research papers addressing basic problems in networked control systems, such as $[5,12]$. In this paper, the system shown in Figure 1 is described by a nonlinear controlled Lipschitz system subject to bounded process and measurement noises over the digital noiseless channel. Lipschitz is a large class of nonlinear systems, such as systems with dead zone and saturation nonlinearities. Furthermore, an important class of linear systems is a special class of Lipschitz systems. The digital noiseless channel is also a basic digital communication channel. 


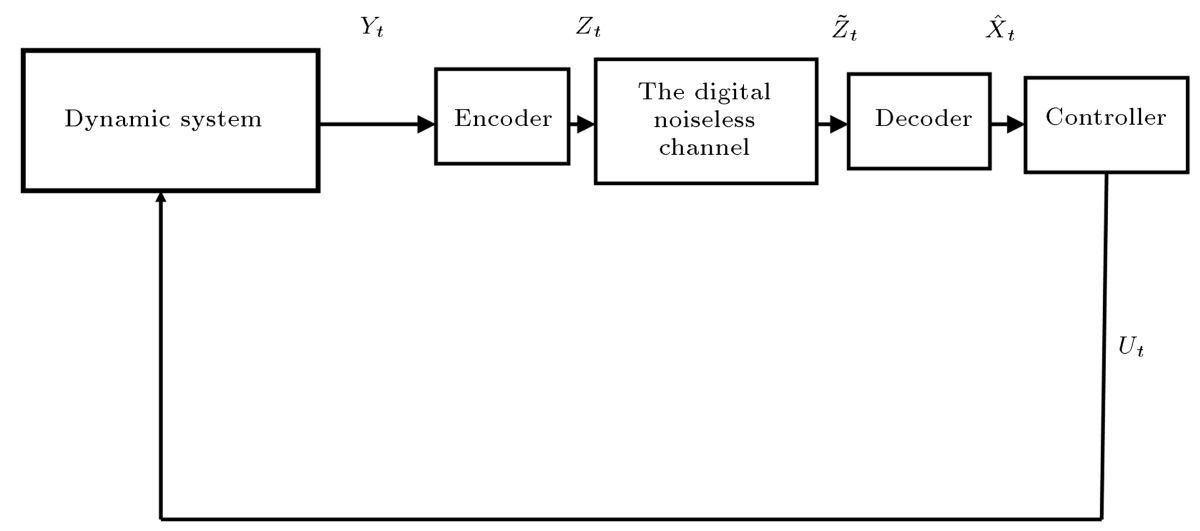

Figure 1. A control system over the digital noiseless channel.

\subsection{Paper contributions}

In this paper, we address the problem of almost sure bounded stability of controlled nonlinear Lipschitz systems subject to bounded process and measurement noises when measurements from dynamic system sampled by sensor are transmitted via the digital noiseless channel to controller (see Figure 1). As the sampled measurements are real-valued, to transmit them over digital links, they must be quantized and represented as a packet of binary data with a specific length (e.g., $R$ bits). This results in distortion in sampled measurements when they are reconstructed at controller. That is, another source of uncertainty considered in this paper is distortion in measurements due to quantization. Despite these uncertainties, a stabilizing technique (which consists of an encoder, decoder, and a controller) and a sufficient condition relating transmission rate, $R$, to Lipschitz coefficients are presented that result in almost sure asymptotic bounded stability. In the absence of process and measurement noises, it is shown that the proposed stabilizing technique results in almost sure asymptotic stability despite distortion in measurements due to quantization. The satisfactory performance of the proposed stabilizing technique for almost sure asymptotic bounded stability and asymptotic stability is also illustrated via computer simulations.

To the best of our knowledge, only the problem of the state estimation of nonlinear uncontrolled Lipschitz dynamic systems over communication channels subject to imperfections (known as tracking) has been addressed in the literature; hence, the main novelty of this paper is the stability of nonlinear noisy controlled Lipschitz systems, in addition to tracking, over the digital noiseless channel. The problem of the state estimation of uncontrolled nonlinear Lipschitz systems over digital links was first considered in [15]. In [15], the authors addressed the problem of the state estimation of a noiseless uncontrolled Lipschitz system over the digital noiseless channel subject to quantization effect. In [16], this result was extended to distributed uncon- trolled noisy Lipschitz systems over the packet erasure network.

\subsection{Paper organization}

The paper is organized as follows: In Section 2, the problem formulation is given. Section 3 is devoted to the stability result. In this section, encoder, decoder, controller, and sufficient condition for almost sure asymptotic bounded stability are presented. Simulation results are given in Section 4. Finally, the paper is concluded in Section 5 by summarizing the main contributions of the paper.

\section{Problem formulation}

Throughout, certain conventions are used: $|\cdot|$ denotes the absolute value, $\log _{2}(\cdot)$ denotes the logarithm of base 2 , and ' $\doteq$ ' means 'by definition is equivalent to'. $[X]_{i}$ means the $i$ th element of the vector $X$ and $\mathbb{R}$ denotes the set of real numbers. Cartesian product is denoted by $\times$ and $A^{\prime}$ denotes the transpose of the matrix $A$. $A^{-1}$ denotes the inverse of the square matrix $A$.

This paper is concerned with almost sure asymptotic bounded stability of nonlinear Lipschitz dynamic systems over the digital noiseless communication channel, as shown in the block diagram of Figure 1. The building blocks of Figure 1 are described below.

Dynamic System: The dynamic system is described by the following discrete time nonlinear Lipschitz system:

$$
\begin{aligned}
& \left\{\begin{array}{l}
X_{t+1}=F\left(X_{t}\right)+B U_{t}+W_{t}, \quad X_{0}=\xi, \\
Y_{t}=X_{t}+V_{t},
\end{array}\right. \\
& t \in \mathbb{N}_{+} \doteq\{0,1,2, \cdots\},
\end{aligned}
$$

where $X_{t} \in \mathbb{R}^{n}$ is the state of the system, $n$ is the number of state variables and it is given, $Y_{t}$ is the observation signal, the random variable $\xi$ is the unknown initial state value, $U_{t} \in \mathbb{R}^{m}$ is the control 
vector, $F(\cdot) \in \mathbb{R}^{n}$ is a nonlinear continuous function with components $f_{i}(\cdot)$, and $B \in \mathbb{R}^{n \times m}$ is such that the matrix $B B^{\prime}$ is invertible. Throughout, it is assumed that the probability measure associated with the initial state $X_{0}$ with components $X_{0}^{(i)}, i=\{1,2, \cdots, n\}$, has bounded support. That is, for each $i=\{1,2, \cdots, n\}$, there exists a compact set $\left[-L_{0}^{(i)}, L_{0}^{(i)}\right] \in \mathbb{R}$ such that $\operatorname{Pr}\left(X_{0}^{(i)} \in\left[-L_{0}^{(i)}, L_{0}^{(i)}\right]\right)=1$. Also, for each $i, f_{i}(\cdot)$ is Lipschitz. That is, for each $f_{i}(\cdot)$, there exists a $K_{i}>0$ (known as Lipschitz coefficient) such that the following inequality holds for all:

$$
\begin{aligned}
& X=\left(\begin{array}{llll}
X^{(1)} & X^{(2)} & \cdots & X^{(n)}
\end{array}\right)^{\prime} \in \mathbb{R}^{n}, \\
& Y=\left(\begin{array}{llll}
Y^{(1)} & Y^{(2)} & \cdots & Y^{(n)}
\end{array}\right)^{\prime} \in \mathbb{R}^{n}, \\
& \left|f_{i}(X)-f_{i}(Y)\right| \leq K_{i}\left(\left|X^{(1)}-Y^{(1)}\right|+\left|X^{(2)}-Y^{(2)}\right|\right. \\
& \left.+\cdots+\left|X^{(n)}-Y^{(n)}\right|\right) \text {. }
\end{aligned}
$$

In the dynamic system (1), $W_{t} \in \mathbb{R}^{n}$ with the components $W_{t}^{(i)}, i=\{1,2, \cdots, n\}$, is the process noise vector and $V_{t} \in \mathbb{R}^{n}$ with the components $V_{t}^{(i)}$, $i=\{1,2, \cdots, n\}$, is the measurement noise vector. Throughout, it is assumed that $W_{t}^{(i)}$ and $V_{t}^{(i)}$ are uniformly distributed random variables with supports $\left[-\omega^{(i)}, \omega^{(i)}\right]$ and $\left[-\gamma^{(i)}, \gamma^{(i)}\right]$, respectively (i.e., $W_{t}^{(i)} \in$ $\left[-\omega^{(i)}, \omega^{(i)}\right]$ and $\left.V_{t}^{(i)} \in\left[-\gamma^{(i)}, \gamma^{(i)}\right]\right)$.

Communication channel: Communication channel between system and controller is the digital noiseless channel with the transmission rate of $R$ bits. This channel transmits a packet of binary data with the rate of $R$ bits in each channel use.

As the sampled measurements are real-valued, to transmit them over the digital noiseless channel, they must be quantized and represented as a packet of binary data with the length of $R$ bits. This is done by the encoder in the block diagram of Figure 1 . On the other hand, the decoder reconstructs the quantized sampled measurements at the receiver. The formal descriptions of the encoder and decoder are given below.

Encoder: Encoder is a causal operator denoted by $Z_{t}=\mathcal{E}\left(Y_{t}\right)$ that maps the system output $Y_{t}$ to channel input $Z_{t}$, which is a string of binaries with the length of $R$ bits.

Decoder: Decoder is a causal operator denoted by $\hat{X}_{t}=\mathcal{D}\left(\tilde{Z}_{t}\right)$ that maps the channel output to $\hat{X}_{t}$ (the estimate of the state variable at the decoder).

Controller: Controller has the following form:

$$
U_{t}=-B^{\prime}\left(B B^{\prime}\right)^{-1} F\left(\hat{X}_{t}\right)
$$

The objective of this paper is to design an encoder, a decoder, and a controller that result in almost sure asymptotic bounded stability of the system (1), as defined below.

Definition 2.1 (almost sure asymptotic bounded stability). Consider the block diagram of Figure 1 described by the nonlinear dynamic system (1) over the digital noiseless channel, as described above. It is said that the system is almost sure asymptotic bounded stabilizable if there exist encoder, decoder, controller, and a closed bounded set $\Delta \subset \mathbb{R}^{n}$ such that the following property holds:

$$
\operatorname{Pr}\left(\lim _{t \rightarrow \infty} X_{t} \in \Delta\right)=1
$$

Remark 2.2. For $\Delta=\{0\}$, we have almost sure asymptotic stability.

\section{Stability result}

In this section, we present a sufficient condition for the transmission rate $R$ such that by using the controller $U_{t}=-B^{\prime}\left(B B^{\prime}\right)^{-1} F\left(\hat{X}_{t}\right)$, the dynamic system (1) in the block diagram of Figure 1 is almost sure asymptotic bounded stable. This result is shown in Theorem 3.1. To obtain this sufficient condition in Theorem 3.1, we construct an encoder and a decoder so that under this condition, the controller $U_{t}=-B^{\prime}\left(B B^{\prime}\right)^{-1} F\left(\hat{X}_{t}\right)$ results in almost sure asymptotic bounded stability.

For simplicity in understanding these encoder and decoder functions, suppose that the system (1) is scalar (i.e., $n=1$ ). At time instant $t=0$, both encoder and decoder divide the interval $\left[-L_{0}^{(1)}-\gamma^{(1)}, L_{0}^{(1)}+\right.$ $\left.\gamma^{(1)}\right]\left(Y_{0}^{(1)} \in\left[-L_{0}^{(1)}-\gamma^{(1)}, L_{0}^{(1)}+\gamma^{(1)}\right]\right)$ into $2^{R}$ equal-size non-overlapping sub-intervals and choose the center of each sub-interval as its index. Now, upon observing $Y_{0}^{(1)}$, encoder determines the sub-interval where $Y_{0}^{(1)}$ is located and represents the index of this sub-interval (denoted by $j$ ) by $R$ bits; then, it transmits these $R$ bits to decoder via the digital noiseless channel. Decoder, after receiving these $R$ bits, determines the index of the sub-interval, where $Y_{0}^{(1)}$ is located (i.e., $j$ ) and outputs $\hat{Y}_{0}^{(1)}\left(=\hat{X}_{0}^{(1)}\right)=j$, where $\hat{Y}_{0}^{(1)}$ is the estimation of $Y_{0}^{(1)}$ and $\hat{X}_{0}^{(1)}$ is the estimation of $X_{0}^{(1)}$ at the end of communication link. Hence, the decoding error for this case is bounded above by $\left|Y_{0}^{(1)}-\hat{Y}_{0}^{(1)}\right| \leq \frac{L_{0}^{(1)}+\gamma^{(1)}}{2^{R}}$.

At time instant $t=1$, encoder computes the error $Y_{1}^{(1)}-\tilde{Y}_{1}^{(1)}$, where $\tilde{Y}_{1}^{(1)}=f_{1}\left(\hat{X}_{0}^{(1)}\right)+B U_{0}\left(U_{0}=\right.$ $\left.-\frac{1}{B} f_{1}\left(\hat{X}_{0}^{(1)}\right)\right)$. Both encoder and decoder divide the interval $Y_{1}^{(1)}-\tilde{Y}_{1}^{(1)} \in\left[-L_{1}^{(1)}-\gamma^{(1)}, L_{1}^{(1)}+\gamma^{(1)}\right]$, where 
$L_{1}^{(1)}=K_{1}\left(\frac{L_{0}^{(1)}+\gamma^{(1)}}{2^{R}}+\gamma^{(1)}\right)+\omega^{(1)}$ in $2^{R}$ sub-intervals; and they repeat the above procedure until decoder outputs $\hat{Y}_{1}^{(1)}\left(=\hat{X}_{1}^{(1)}\right)=j+\tilde{Y}_{1}^{(1)}$. As a result, the decoding error is bounded above by $\left|Y_{1}^{(1)}-\hat{Y}_{1}^{(1)}\right| \leq$ $\frac{L_{1}^{(1)}+\gamma^{(1)}}{2^{R}}$.

By following the above procedure, $\hat{X}_{0}^{(1)}, \hat{X}_{1}^{(1)}$, $\hat{X}_{2}^{(1)}, \cdots$ are constructed and decoding error is bounded above by $\left|Y_{t}^{(1)}-\hat{Y}_{t}^{(1)}\right| \leq \frac{L_{t}^{(1)}+\gamma^{(1)}}{2^{R}}$, where:

$$
L_{t}^{(1)}=\frac{K_{1}}{2^{R}} L_{t-1}^{(1)}+\frac{K_{1} \gamma^{(1)}}{2^{R}}+K_{1} \gamma^{(1)}+\omega^{(1)} .
$$

Now, under the assumption of $\left|\frac{K_{1}}{2^{R}}\right|<1$, the above dynamic system for $L_{t}^{(1)}$ is stable; hence, $L_{t}^{(1)}$ is asymptotically bounded. Therefore, under this assumption, using the above encoding and decoding technique, tracking of $Y_{t}^{(1)}$ by $\hat{Y}_{t}^{(1)}$ with bounded error at the end of communication is achieved.

Now, after this instruction and simple explanation of the designed method and algorithm, we are ready to present the main theorem of this paper.

Theorem 3.1. Consider the control system of Figure 1 described by the nonlinear uncertain Lipschitz system (1) over the digital noiseless channel, as described earlier. Suppose that there exist non-negative integers $R_{1}, R_{2}, \cdots, R_{n}$ that make the following matrix stable:

$$
\mathcal{A} \doteq\left(\begin{array}{cccc}
\frac{K_{1}}{2^{R_{1}}} & \frac{K_{1}}{2^{R_{2}}} & \cdots & \frac{K_{1}}{2^{R_{n}}} \\
\frac{K_{2}}{2^{R_{1}}} & \frac{K_{2}}{2^{R_{2}}} & \cdots & \frac{K_{2}}{2^{R_{n}}} \\
\vdots & & & \\
\frac{K_{n}}{2^{R_{1}}} & \frac{K_{n}}{2^{R_{2}}} & \cdots & \frac{K_{n}}{2^{R_{n}}}
\end{array}\right) .
$$

Then, using the control policy $U_{t}=-B^{\prime}\left(B B^{\prime}\right)^{-1} F$ $\left(\hat{X}_{t}\right)$, there exists a closed bounded set $\Delta \subset \mathbb{R}^{n}$ such that $X_{t} \rightarrow \Delta$, P-a.s.; or equivalently, $\operatorname{Pr}\left(\lim _{t \rightarrow \infty} X_{t} \in\right.$ $\Delta)=1$, where:

$$
\begin{aligned}
& \Delta=\left[-L_{\infty}^{(1)}, L_{\infty}^{(1)}\right] \times\left[-L_{\infty}^{(2)}, L_{\infty}^{(2)}\right] \times \cdots \times\left[-L_{\infty}^{(n)}, L_{\infty}^{(n)}\right], \\
& L_{\infty}^{(i)} \doteq\left[\lim _{t \rightarrow \infty} \sum_{j=0}^{t-1} \mathcal{A}^{t-1-j}((\mathcal{A}+\mathcal{B}) \gamma+\omega)\right]_{i} .
\end{aligned}
$$

Proof. To prove this theorem, we show that the extended version of the above encoding and decoding technique applied to the system (1) with $n$ states along with the controller $U_{t}=-B^{\prime}\left(B B^{\prime}\right)^{-1} F\left(\hat{X}_{t}\right)$ results in almost sure asymptotic bounded stability if matrix (2) is stable.
Encoding and decoding technique: At time instant $t=0$ for each $i=\{1,2, \cdots, n\}$, the set $\left[-L_{0}^{(i)}-\gamma^{(i)}, L_{0}^{(i)}+\gamma^{(i)}\right]$ is partitioned into $2^{R_{i}}$ equalsize, non-overlapping sub-intervals and the center of each sub-interval is chosen as its index. For each $i=\{1,2, \cdots, n\}$, upon observing $Y_{0}\left(=X_{0}+V_{0}\right)$, the index of the sub-interval that includes $Y_{0}^{(i)}$ is encoded into $R_{i}$ bits. Then, a packet with the length of $R=$ $R_{1}+R_{2}+\cdots+R_{n}$ bits containing information about the initial measurement $Y_{0}$ is transmitted via the channel. When the decoder receives these $R$ bits, for each $i$, it identifies the index of the sub-interval where $Y_{0}^{(i)}$ is located; and the value of this index is chosen as $\hat{Y}_{0}^{(i)}=\hat{X}_{0}^{(i)}$ (the estimation of $Y_{0}^{(i)}$ and $X_{0}^{(i)}$ at the receiver). Therefore, the estimation error is bounded above by $\left|X_{0}^{(i)}-\hat{X}_{0}^{(i)}\right| \leq \frac{L_{0}^{(i)}+\gamma^{(i)}}{2^{R_{i}}}+\gamma^{(i)}$.

At time instant $t=1$, from the Lipschitz continuity assumption, for each $i=\{1,2, \cdots, n\}$, an upper bound on $X_{1}^{(i)}$ is calculated as follows:

$$
\begin{aligned}
\left|X_{1}^{(i)}\right| & =\left|f_{i}\left(X_{0}\right)+\left[B U_{0}\right]_{i}+W_{0}^{(i)}\right| \\
& =\left|f_{i}\left(X_{0}\right)-f_{i}\left(\hat{X}_{0}\right)+W_{0}^{(i)}\right| \\
& \leq K_{i}\left(\left|X_{0}^{(1)}-\hat{X}_{0}^{(1)}\right|+\cdots+\left|X_{0}^{(n)}-\hat{X}_{0}^{(n)}\right|\right)+\omega^{(i)} \\
& =\omega^{(i)}+K_{i} \sum_{j=1}^{n}\left(\frac{L_{0}^{(j)}+\gamma^{(j)}}{2^{R_{j}}}+\gamma^{(j)}\right) \doteq L_{1}^{(i)} .
\end{aligned}
$$

Then, similar to the previous time instant, at this time instant, for each $i=\{1,2, \cdots, n\}$, the interval $\left[-L_{1}^{(i)}-\gamma^{(i)}, L_{1}^{(i)}+\gamma^{(i)}\right]$ is partitioned into $2^{R_{i}}$ equalsize, non-overlapping sub-intervals and the center of each sub-interval is chosen as its index. Having that, for each $i$, upon observing $Y_{1}\left(=X_{1}+V_{1}\right)$, the index of the sub-interval that includes $Y_{1}^{(i)}$ is encoded into $R_{i}$ bits. Then, $R=R_{1}+R_{2}+\cdots+R_{n}$ bits containing information about $Y_{1}$ is transmitted via the channel. When these $R$ bits are received, for each $i$, the decoder identifies the index of the sub-interval that contains $Y_{1}^{(i)}$; and the value of this index is chosen as $\hat{Y}_{1}^{(i)}=$ $\hat{X}_{1}^{(i)}$. Therefore, the estimation error is bounded above by $\left|X_{1}^{(i)}-\hat{X}_{1}^{(i)}\right| \leq \frac{L_{1}^{(i)}+\gamma^{(i)}}{2^{R_{i}}}+\gamma^{(i)}$.

By following a similar procedure as described above, the sequence $\hat{X}_{0}, \hat{X}_{1}, \hat{X}_{2}, \hat{X}_{3}, \cdots$ is constructed at the decoder.

Now, we must show that by using the above coding technique and controller $U_{t}=-B^{\prime}\left(B B^{\prime}\right)^{-1} F\left(\hat{X}_{t}\right)$ there exists a closed and bounded set $\Delta \subset \mathbb{R}^{n}$ such that $\operatorname{Pr}\left(\lim _{t \rightarrow \infty} X_{t} \in \Delta\right)=1$ provided that Eq. (2) is stable. To achieve this goal, choose any rates $R_{1}, R_{2}, \cdots, R_{n}$ that make matrix $\mathcal{A}$ stable. Now, using the above 
encoding and decoding technique and the controller $U_{t}=-B^{\prime}\left(B B^{\prime}\right)^{-1} F\left(\hat{X}_{t}\right)$, we have:

$$
\begin{aligned}
& \left|X_{0}^{(i)}\right| \leq L_{0}^{(i)}, \\
& \left|X_{1}^{(i)}\right| \leq \omega^{(i)}+K_{i} \sum_{j=1}^{n}\left(\frac{L_{0}^{(j)}+\gamma^{(j)}}{2^{R_{j}}}+\gamma^{(j)}\right) \doteq L_{1}^{(i)} \\
& \left|X_{2}^{(i)}\right| \leq \omega^{(i)}+K_{i} \sum_{j=1}^{n}\left(\frac{L_{1}^{(j)}+\gamma^{(j)}}{2^{R_{j}}}+\gamma^{(j)}\right) \doteq L_{2}^{(i)} \\
& \vdots \\
& \left|X_{t}^{(i)}\right| \leq \omega^{(i)}+K_{i} \sum_{j=1}^{n}\left(\frac{L_{t-1}^{(j)}+\gamma^{(j)}}{2^{R_{j}}}+\gamma^{(j)}\right) \doteq L_{t}^{(i)} .
\end{aligned}
$$

Now, let:

$$
Z_{t} \doteq\left(\begin{array}{c}
L_{t}^{(1)} \\
L_{t}^{(2)} \\
\vdots \\
L_{t}^{(n)}
\end{array}\right) .
$$

Then, from the recursive equation:

$$
L_{t}^{(i)}=\omega^{(i)}+K_{i} \sum_{j=1}^{n}\left(\frac{L_{t-1}^{(j)}+\gamma^{(j)}}{2^{R_{j}}}+\gamma^{(j)}\right),
$$

which almost surely defines an upper bound on $X_{t}^{(i)}$, we have the following dynamic model for the vector $Z_{t}$ :

$$
Z_{t+1}=\mathcal{A} Z_{t}+(\mathcal{A}+\mathcal{B}) \gamma+\omega
$$

where:

$$
\begin{aligned}
Z_{0}=\left(\begin{array}{c}
L_{0}^{(1)} \\
L_{0}^{(2)} \\
\vdots \\
L_{0}^{(n)}
\end{array}\right), & \mathcal{B} \doteq\left(\begin{array}{cccc}
K_{1} & K_{1} & \cdots & K_{1} \\
K_{2} & K_{2} & \cdots & K_{2} \\
\vdots & & & \\
K_{n} & K_{n} & \cdots & K_{n}
\end{array}\right), \\
\gamma \doteq\left(\begin{array}{c}
\gamma^{(1)} \\
\gamma^{(2)} \\
\vdots \\
\gamma^{(n)}
\end{array}\right), & \omega \doteq\left(\begin{array}{c}
\omega^{(1)} \\
\omega^{(2)} \\
\vdots \\
\omega^{(n)}
\end{array}\right) .
\end{aligned}
$$

Now, from the well-known stability results of linear time-invariant systems, for the linear time-invariant dynamic system (3), it follows that all components of the vector $Z_{t}$ are asymptotically bounded if and only if matrix $\mathcal{A}$ is stable. Hence, as we chose the rates $R_{1}, R_{2}, \cdots, R_{n}$ such that matrix $\mathcal{A}$ is stable and as $Z_{t}^{(i)}$ almost surely defines an upper bound on $X_{t}^{(i)}$, the dynamic system (1) is almost sure asymptotic bounded stable as follows:

$$
X_{t} \rightarrow \Delta, \text { P-a.s., }
$$

where:

$$
\begin{aligned}
& \Delta=\left[-L_{\infty}^{(1)}, L_{\infty}^{(1)}\right] \times\left[-L_{\infty}^{(2)}, L_{\infty}^{(2)}\right] \times \cdots \times\left[-L_{\infty}^{(n)}, L_{\infty}^{(n)}\right], \\
& L_{\infty}^{(i)}=\left[\lim _{t \rightarrow \infty} \sum_{j=0}^{t-1} \mathcal{A}^{t-1-j}((\mathcal{A}+\mathcal{B}) \gamma+\omega)\right]_{i} .
\end{aligned}
$$

We have the following 2 propositions as a direct result of the above theorem.

Proposition 3.2. For the noiseless dynamic system, i.e., $\gamma^{(i)}=\omega^{(i)}=0$, we have almost sure asymptotic stability as $X_{t} \rightarrow 0$, P-a.s., provided that the rates $R_{1}, R_{2}, \cdots, R_{n}$ make matrix $\mathcal{A}$ stable.

Proof. As the rates $R_{1}, R_{2}, \cdots, R_{n}$ make matrix $\mathcal{A}$ stable, from Theorem 3.1, it follows that $X_{t} \rightarrow \Delta$, Pa.s., where:

$$
\begin{aligned}
& \Delta=\left[-L_{\infty}^{(1)}, L_{\infty}^{(1)}\right] \times\left[-L_{\infty}^{(2)}, L_{\infty}^{(2)}\right] \times \cdots \times\left[-L_{\infty}^{(n)}, L_{\infty}^{(n)}\right], \\
& L_{\infty}^{(i)}=\left[\lim _{t \rightarrow \infty} \sum_{j=0}^{t-1} \mathcal{A}^{t-1-j}((\mathcal{A}+\mathcal{B}) \gamma+\omega)\right]_{i}
\end{aligned}
$$

Now, as for each $i=\{1,2, \cdots, n\}$, it is assumed that $\gamma^{(i)}=\omega^{(i)}=0$. We have:

$$
\sum_{j=0}^{t-1} \mathcal{A}^{t-1-j}((\mathcal{A}+\mathcal{B}) \gamma+\omega)=0,
$$

hence, $X_{t} \rightarrow 0$, P-a.s.

Proposition 3.3. Consider the control system of Figure 1 described by the scalar version of the nonlinear uncertain Lipschitz system (1) over the digital noiseless channel with rate $R>\log _{2} K_{1}$, where $K_{1}>0$ is the Lipschitz coefficient (i.e., $\left|f_{1}(X)-f_{1}(Y)\right| \leq K_{1}|X-Y|$, $\forall X, Y \in \mathbb{R})$. Then, using the proposed encoding and decoding technique and $U_{t}=-\frac{1}{B} f_{1}\left(\hat{X}_{t}^{(1)}\right)$, there exists the set:

$$
\begin{aligned}
\Delta= & {\left[-\left(\left(\frac{K_{1}}{2^{R}}+K_{1}\right) \gamma^{(1)}+\omega^{(1)}\right) \frac{1}{1-\frac{K_{1}}{2^{R}}},\left(\left(\frac{K_{1}}{2^{R}}\right.\right.\right.} \\
& \left.\left.\left.+K_{1}\right) \gamma^{(1)}+\omega^{(1)}\right) \frac{1}{1-\frac{K_{1}}{2^{R}}}\right]
\end{aligned}
$$

such that $X_{t}^{(1)} \rightarrow \Delta$ P-a.s.; or equivalently, $\operatorname{Pr}\left(\lim _{t \rightarrow \infty} X_{t}^{(1)} \in \Delta\right)=1$. 
Proof. For the scalar system, matrix $\mathcal{A}$ is reduced to $\mathcal{A}=\left(\frac{K_{1}}{2^{R}}\right)$, which is stable for any rate $R>\log _{2} K_{1}$. Hence, as we assumed that $R>\log _{2} K_{1}$, it follows from Theorem 3.1 that $X_{t} \rightarrow \Delta$, P-a.s., where:

$$
\begin{aligned}
\Delta= & {\left[-L_{\infty}^{(1)}, L_{\infty}^{(1)}\right] } \\
L_{\infty}^{(1)} & =\lim _{t \rightarrow \infty} \sum_{j=0}^{t-1} \mathcal{A}^{t-1-j}\left((\mathcal{A}+\mathcal{B}) \gamma^{(1)}+\omega^{(1)}\right) \\
& =\left(\left(\frac{K_{1}}{2^{R}}+K_{1}\right) \gamma^{(1)}+\omega^{(1)}\right) \frac{1}{1-\frac{K_{1}}{2^{R}}}
\end{aligned}
$$

This completes the proof.

Remark 3.4. We have the following remarks regarding the above results:

i) From the specific structure of matrix $\mathcal{A}$, it follows that the eigenvalues of this matrix are: $0,0, \cdots, 0, \frac{K_{1}}{2^{R 1}}+\frac{K_{2}}{2^{R_{2}}}+\cdots+\frac{K_{n}}{2^{R_{n}}}$. Hence, a sufficient condition on the rates $R_{i} \mathrm{~s}$ for the stability using the proposed stabilizing technique is the following:

$$
\begin{gathered}
R_{i}>\max \left\{0, \log _{2} K_{i}\right\}, \\
\forall i \in\{1,2, \cdots, n\}
\end{gathered}
$$

ii) In general, the weaker condition:

$$
R \geq \sum_{i ; K_{i}>1} \log _{2} K_{i}
$$

does not imply the stronger condition (4). However, for those cases that the weaker condition (5) implies the stronger condition (4) (e.g., the case for $K_{1}=5$ and $K_{2}=7$ ), we can conclude that condition (5) is also a sufficient condition for stability.

iii) For linear time-invariant noiseless systems with eigenvalues $\lambda_{i}(A) \mathrm{s}$ ( $A$ is the system matrix) over the packet erasure channel with the rate of $R$ bits (which includes the digital noiseless channel as a special case), it is shown in [17] that condition (6) on the rates of $R_{1}, R_{2}, \cdots, R_{n}\left(R=R_{1}+R_{2}+\right.$ $\left.\cdots+R_{n}\right)$ is a sufficient condition for almost sure asymptotic stability:

$$
\begin{gathered}
R_{i}>\max \left\{0, \log _{2}\left|\lambda_{i}(A)\right|\right\}, \\
\forall i \in\{1,2, \cdots, n\} .
\end{gathered}
$$

Furthermore, independent of the choice of encoder, decoder, and controller, the following condition, known as the eigenvalues rate condition, is necessary for almost sure asymptotic stability:

$$
R \geq \sum_{i ;\left|\lambda_{i}(A)\right|>1} \log _{2}\left|\lambda_{i}(A)\right| .
$$

In general, the eigenvalues rate condition does not imply the stronger condition (6). But, for those cases that the eigenvalues rate condition implies the stronger condition (6) (e.g., the case for the system matrix $A=\left(\begin{array}{cc}11 & 8 \\ -3 & 1\end{array}\right)$ ), in [17] it is concluded that the eigenvalues rate condition $(7)$ is a necessary and sufficient condition (a tight bound on transmission rate $R$ ) for almost sure asymptotic stability of linear time-invariant noiseless systems over the packet erasure channel.

iv) From the above remarks, it follows that for linear time-invariant noiseless systems over the digital noiseless channel, condition (5) is a necessary and sufficient condition (a tight bound on transmission rate) for almost sure asymptotic stability if $K_{i}=$ $\left|\lambda_{i}(A)\right|$ and the eigenvalues rate condition implies the stronger condition $(6)$.

To the best of our knowledge, similar works to this work were previously reported in $[15,16]$ where they only addressed the problem of tracking states of uncontrolled Lipschitz systems over the digital noiseless and the packet erasure channels, respectively. Note that the digital noiseless channel is a special case of packet erasure channel when erasure probability is zero. In [15], the authors considered noiseless uncontrolled Lipschitz systems and presented a sufficient condition for mean square asymptotic tracking in which, for the scalar system, this condition was reduced to the condition found in this paper for tracking (i.e., $\left.\left|\frac{K_{1}}{2^{R}}\right|<1\right)$. In [16], the authors addressed the problem of tracking of a distributed system of uncontrolled Lipschitz distributed noisy sub-systems over the packet erasure network. For mean absolute tracking, which is a weaker notion for tracking than the almost sure notion used in this paper, they found a sufficient condition, which, for the special case of single subsystem, was reduced to the condition found in this paper.

\section{Simulation results}

In this section, we illustrate the satisfactory performance of the proposed encoder, decoder, and controller for almost sure asymptotic bounded stability and asymptotic stability using computer simulations.

Define the nonlinear Lipschitz functions $\operatorname{sat}(\cdot)$ and $\operatorname{deadz}(\cdot)$ as follows: 


$$
\begin{aligned}
& \operatorname{sat}(x) \doteq \begin{cases}30, & x \geq 10 \\
3 x, & -10<x<10 \\
-30, & x \leq-10\end{cases} \\
& \operatorname{deadz}(x) \doteq \begin{cases}2(x-1), & x \geq 1 \\
0, & -1<x<1 \\
2(x+1), & x \leq-1\end{cases}
\end{aligned}
$$

Now, suppose the control system of Figure 1 is described by the following coupled nonlinear system.

$$
\left\{\begin{array}{l}
X_{t+1}^{(1)}=\operatorname{dead} z\left(X_{t}^{(1)}+3 X_{t}^{(2)}\right)+U_{t}^{(1)}+W_{t}^{(1)} \\
Y_{t}^{(1)}=X_{t}^{(1)}+V_{t}^{(1)} \\
X_{t+1}^{(2)}=\operatorname{sat}\left(2 X_{t}^{(1)}+X_{t}^{(2)}\right)+U_{t}^{(2)}+W_{t}^{(2)} \\
Y_{t}^{(2)}=X_{t}^{(2)}+V_{t}^{(2)}
\end{array}\right.
$$

Here, $X_{0}^{(1)}$ and $X_{0}^{(2)} \in[-20,20]$ are unknown initial states and $W_{t}^{(i)}$ and $V_{t}^{(i)}$ are uniformly distributed random variables with the support $[-0.1,0.1]$ (i.e., $\left.W_{t}^{(i)}, V_{t}^{(i)} \in[-0.1,0.1]\right)$.

Figure 2 illustrates the state trajectories of system (8) when $U_{t}^{(1)}=U_{t}^{(2)}=0$. As is clear from Figure 2, without control inputs, the system is unstable. To stabilize this system, the control vector is set to:

$$
\begin{aligned}
& U_{t}=\left(\begin{array}{l}
U_{t}^{(1)} \\
U_{t}^{(2)}
\end{array}\right), \\
& U_{t}^{(1)}=-\operatorname{deadz}\left(\hat{X}_{t}^{(1)}+3 \hat{X}_{t}^{(2)}\right), \\
& U_{t}^{(2)}=-\operatorname{sat}\left(2 \hat{X}_{t}^{(1)}+\hat{X}_{t}^{(2)}\right) .
\end{aligned}
$$

For this system, the Lipschitz coefficients $K_{1}$, and $K_{2}$ are determined as follows:

$$
\begin{aligned}
\mid \operatorname{deadz} & \left(X^{(1)}+3 X^{(2)}\right)-\operatorname{deadz}\left(Y^{(1)}+3 Y^{(2)}\right) \mid \\
& \leq 2\left|X^{(1)}+3 X^{(2)}-Y^{(1)}-3 Y^{(2)}\right| \\
& =2\left|\left(X^{(1)}-Y^{(1)}\right)+3\left(X^{(2)}-Y^{(2)}\right)\right| \\
& \leq 2\left|X^{(1)}-Y^{(1)}\right|+6\left|X^{(2)}-Y^{(2)}\right| \\
& \leq 6\left(\left|X^{(1)}-Y^{(1)}\right|+\left|X^{(2)}-Y^{(2)}\right|\right) .
\end{aligned}
$$

Hence, $K_{1}=6$. Note that the first inequality above follows from the definition of $\operatorname{dead} z($.$) . Similarly, for$ $K_{2}$, we have:
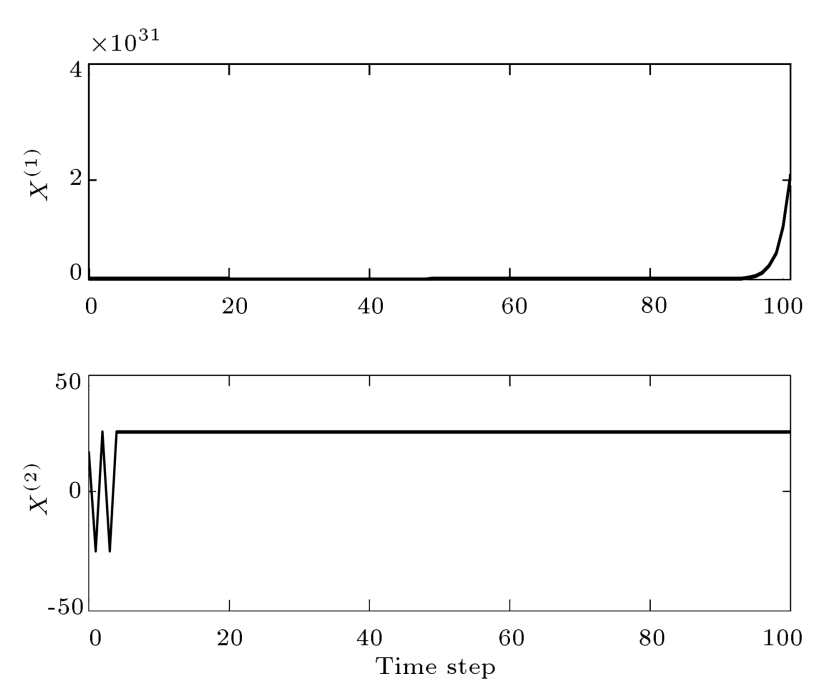

Figure 2. The state trajectories without control inputs.

$$
\begin{aligned}
& \mid \text { sat }\left(2 X^{(1)}+X^{(2)}\right)-\operatorname{sat}\left(2 Y^{(1)}+Y^{(2)}\right) \mid \\
& \quad \leq 3\left|2 X^{(1)}+X^{(2)}-2 Y^{(1)}-Y^{(2)}\right| \\
& \quad=3\left|2\left(X^{(1)}-Y^{(1)}\right)+\left(X^{(2)}-Y^{(2)}\right)\right| \\
& \quad \leq 6\left|X^{(1)}-Y^{(1)}\right|+3\left|X^{(2)}-Y^{(2)}\right| \\
& \quad \leq 6\left(\left|X^{(1)}-Y^{(1)}\right|+\left|X^{(2)}-Y^{(2)}\right|\right) .
\end{aligned}
$$

Hence, $K_{2}=6$.

Consequently, from Theorem 3.1, it follows that $\mathcal{A}=\left(\begin{array}{cc}\frac{6}{2^{R_{1}}} & \frac{6}{2^{R_{2}}} \\ \frac{2^{R_{1}}}{2^{R_{2}}} & \frac{2^{R_{2}}}{}\end{array}\right) ;$ and as the eigenvalues of the matrix $\mathcal{A}$ are 0 and $6\left(\frac{1}{2^{R_{1}}}+\frac{1}{2^{R_{2}}}\right)$, the rates $\left(R_{1}, R_{2}\right)$ that make matrix $\mathcal{A}$ stable while the transmission rate $R=R_{1}+R_{2}$ is minimum are $\left(R_{1}, R_{2}\right)=(3,5),(5,3)$, $(4,4)$ in which, for this system, we choose $\left(R_{1}, R_{2}\right)=$ $(4,4)$. For these rates, $L_{\infty}^{(i)}, i=1,2$, are calculated as follows:

$$
\begin{aligned}
\sum_{j=0}^{t-1} \mathcal{A}^{t-1-j}((\mathcal{A}+\mathcal{B}) \gamma+\omega) & \\
= & (\mathcal{A}+\mathcal{B}) \gamma+\omega+\sum_{j=0}^{t-2} \mathcal{A}^{t-1-j}((\mathcal{A}+\mathcal{B}) \gamma+\omega) \\
& =\left(\begin{array}{c}
\frac{11}{8} \\
\frac{11}{8}
\end{array}\right)+\sum_{j=0}^{t-2}\left(\frac{3}{8}\right)^{t-1-j}\left(\begin{array}{ll}
2^{t-1-j-1} & 2^{t-1-j-1} \\
2^{t-1-j-1} & 2^{t-1-j-1}
\end{array}\right)\left(\begin{array}{c}
\frac{11}{8} \\
\frac{11}{8}
\end{array}\right) \\
& =\left(\begin{array}{c}
E_{t} \\
E_{t}
\end{array}\right),
\end{aligned}
$$



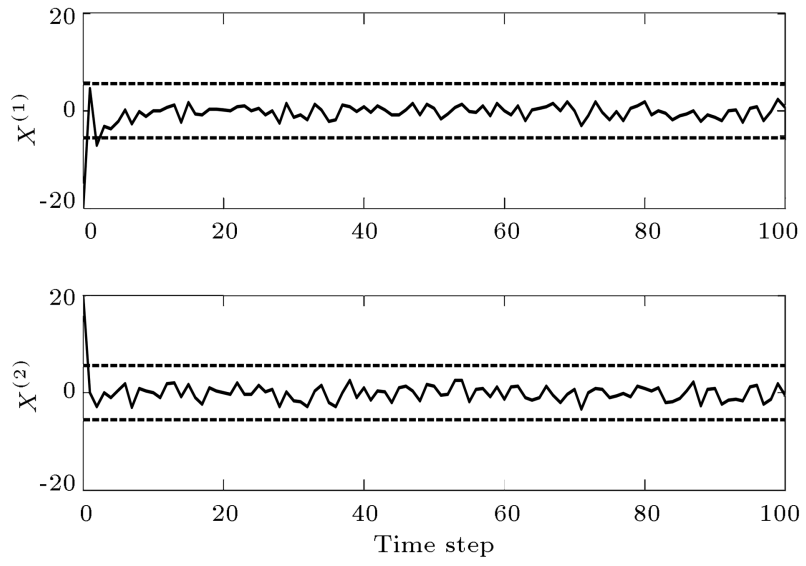

Figure 3. The state trajectories for $\left(R_{1}, R_{2}\right)=(4,4)$. Dashed lines identify the boundaries of the interval $\left[\frac{-44}{8}, \frac{44}{8}\right]$.
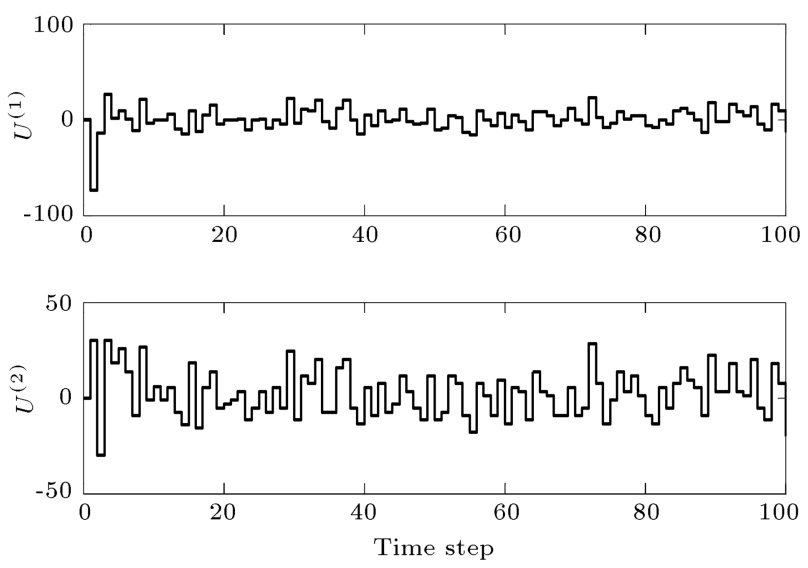

Figure 4. The control trajectories for $\left(R_{1}, R_{2}\right)=(4,4)$.

$$
\begin{aligned}
E_{t} & \doteq \frac{11}{8}+\sum_{j=0}^{t-2}\left(\frac{3}{8}\right)^{t-1-j} \frac{11}{4} 2^{t-1-j-1} \\
& =\frac{11}{8}+\frac{11}{8} 3\left(1-\left(\frac{3}{4}\right)^{t-1}\right)
\end{aligned}
$$

and hence, $L_{\infty}^{(i)}=\lim _{t \rightarrow \infty} E_{t}=\frac{44}{8}$.

Figure 3 illustrates the state trajectories of system (8) and Figure 4 illustrates the control trajectories when the proposed encoder, decoder, and controller are used with rates $\left(R_{1}, R_{2}\right)=(4,4)(R=8$ bits $)$. As is clear from Figure 3 , by increasing time, the state trajectories of the system enter the interval $\left[-\frac{44}{8}, \frac{44}{8}\right]$ and stay there despite uncertainties in dynamic model and distortion due to quantization.

We can shrink the close bounded set $\Delta \subset \mathbb{R}^{2}$ by choosing larger rates. For example, for $\left(R_{1}, R_{2}\right)=$ $(6,6), L_{\infty}^{(i)}=1.6231, i=1,2$. Figure 5 illustrates the state trajectories for this case.

Figure 6 illustrates the state trajectories of the
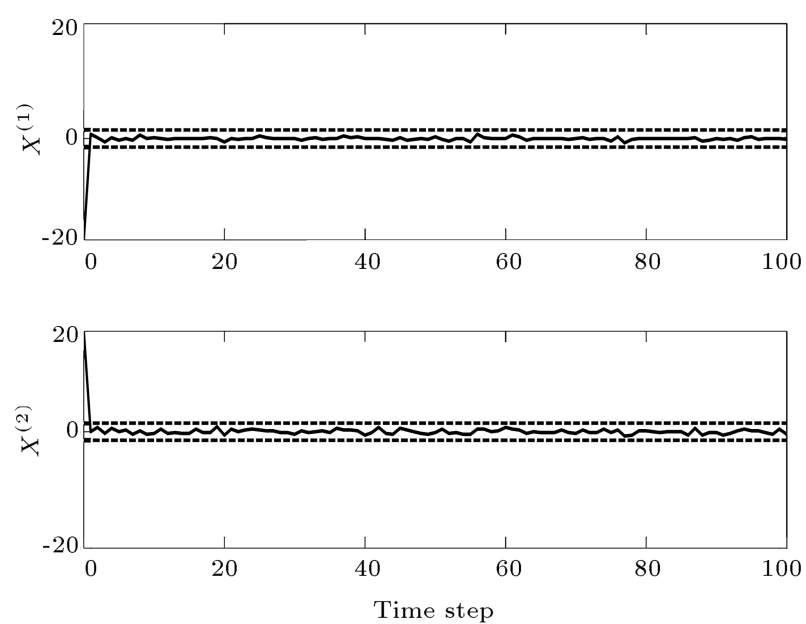

Figure 5. The state trajectories for $\left(R_{1}, R_{2}\right)=(6,6)$. Dashed lines identify the boundaries of the interval $[-1.6231,1.6231]$.
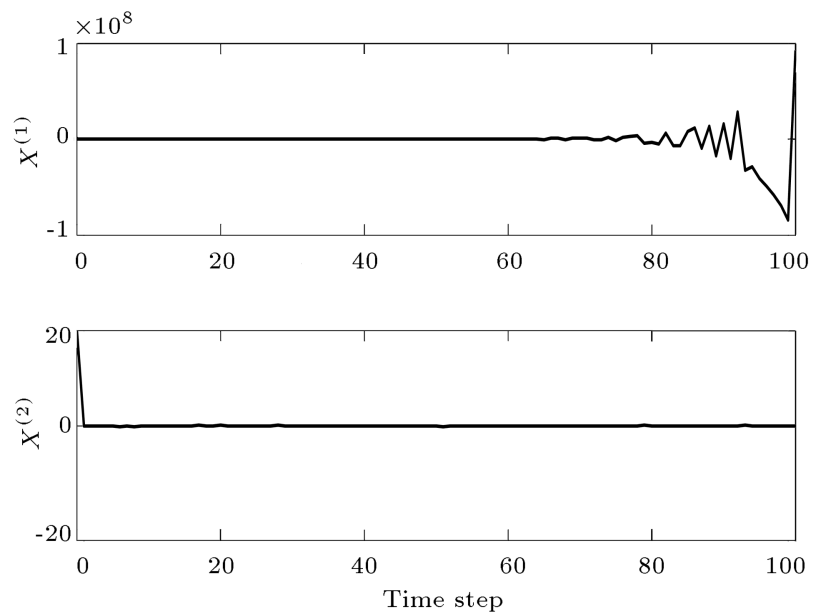

Figure 6. The state trajectories for $\left(R_{1}, R_{2}\right)=(4,3)$.

system when the proposed encoder, decoder, and controller with rates $\left(R_{1}, R_{2}\right)=(4,3)(R=7$ bits $)$ are used. As is clear from Figure 6, for these rates, the proposed stabilizing technique is not able to stabilize the system. This result is expected as the rates $\left(R_{1}, R_{2}\right)=(4,3)$ do not make matrix $\mathcal{A}$ stable.

Figure 7 illustrates the state trajectories of the system when $W_{t}^{(i)}=V_{t}^{(i)}=0, i=\{1,2\}$ and $\left(R_{1}, R_{2}\right)=(4,4)$. As is clear from Figure 7 , by increasing time, the state trajectories, as expected from Proposition 3.2, converge to zero.

\section{Conclusion}

This paper was concerned with the stability of nonlinear Lipschitz systems subject to bounded process and measurement noises when transmission from sensor to controller was subject to quantization distortion. A stabilizing technique and a sufficient condition relating 

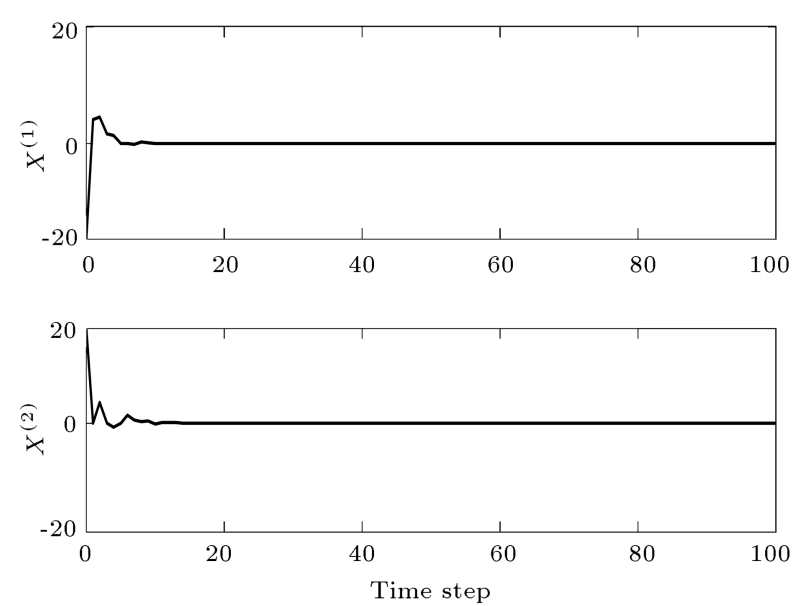

Figure 7. The state trajectories for $\left(R_{1}, R_{2}\right)=(4,4)$ and $W_{t}^{(i)}=V_{t}^{(i)}=0$.

transmission rate $R$ to Lipschitz coefficients $K_{i}$ s were presented for almost sure asymptotic bounded stability of nonlinear uncertain Lipschitz systems. It was shown that in the absence of process and measurement noises, the proposed stabilizing technique resulted in almost sure asymptotic stability.

Furthermore, it was illustrated via computer simulations that the proposed stabilizing technique had satisfactory performance for almost sure asymptotic bounded stability and asymptotic stability.

For future, it is also interesting to consider the effects of random packet dropout in transmission from sensor to controller on almost sure asymptotic bounded stability of nonlinear uncertain Lipschitz systems. It is also interesting to relax the assumption made on matrix $B$

\section{References}

1. Canudas-de-Wit, C., Rubio, F.R., and Corchero, A. "A new mechanism for controlling stick-slip oscillations in oil well drill strings", IEEE Transactions on Control Systems Technology, 16(6), pp. 1177-1191 (2008).

2. Memarzadeh, A. "Optimal borehole communication using multicarrier modulation", Ph.D. Thesis, Rice University (2008).

3. Elia, N. "When Bode meets Shannon: control-oriented feedback communication schemes", IEEE Trans. Automat. Contr., 49(9), pp. 1477-1488 (2004).

4. Elia, N. and Eisenbeis, J.N. "Limitations of linear control over packet drop networks", IEEE Trans. Automat. Contr., 56(4), pp. 826-841 (2011).

5. Martins, N.C., Dahleh, A., and Elia, N. "Feedback stabilization of uncertain systems in the presence of a direct link", IEEE Trans. Automat. Contr., 51(3), pp. $438-447$ (2006).

6. Minero, P., Franceschetti, M., Dey, S. and Nair, N. "Data rate theorem for stabilization over time-varying feedback channels", IEEE Trans. Automat. Contr., 54(2), pp. 243-255 (2009).

7. Minero, P., Coviello, L., and Franceschetti, M. "Stabilization over Markov feedback channels: the general case", IEEE Trans. Automat. Contr., 58(2), pp. 349$362(2013)$.

8. Farhadi, A. "Stability of linear dynamic systems controlled over the packet erasure channel: a co-design approach", International Journal of Control, 88(12), pp. 2488-2498 (2015).

9. Farhadi, A. "Feedback channel in linear noiseless dynamic systems controlled over the packet erasure network", International Journal of Control, 88(8), pp. 1490-1503 (2015).

10. Farhadi, A., Domun, J. and Canudas de Wit, C. "A supervisory control policy over an acoustic communication network", International Journal of Control, 88(5), pp. $946-958$ (2015).

11. Niu, Y. and Ho, D.W.C. "Control strategy with adaptive quantizer's parameters under digital communication channels", Automatica, 50(10), pp. 2665-2671 (2014).

12. Nair, G.N., Evans, R.J., Mareels, I.M.Y., and Moran, W. "Topological feedback entropy and nonlinear stabilization", IEEE Trans. Automat. Contr., 49(9), pp. 1585-1597 (2004).

13. Nair, G.N. and Evans, R.J. "Stabilizability of stochastic linear systems with finite feedback data rates", SIAM J. Control Optimization, 43(3), pp. 413-436 (2004).

14. Canudas de Wit, C., Gomez-Estern, F. and Rodrigues Rubio, F. "Delta-modulation coding redesign for feedback-controlled systems", IEEE Transactions on Industrial Electronics, 56(7), pp. 2684-2696 (2009).

15. Nair, G.N. and Evans, R.J. "State estimation via a capacity limited communication channel", IEEE Conf. Decision Contr., pp. 866-871 (1997).

16. Farhadi, A. and Ahmed, N.U. "Tracking nonlinear noisy dynamic systems over noisy communication channels", IEEE Transactions on Communications, 59(4), pp. 955-961 (2011).

17. Tatikonda, S. and Mitter, S. "Control over noisy channels", IEEE Transactions on Automatic Control, 49(7), pp. 1196-1201 (2004).

\section{Biography}

Alireza Farhadi received the $\mathrm{PhD}$ degree in Electrical Engineering from the University of Ottawa, Ontario, Canada, in 2007. After receiving $\mathrm{PhD}$ degree, Dr. Farhadi worked as Postdoctoral Fellow in the Department of Electrical Engineering of the University of Ottawa during 2008-2009 and the French National Institute for Research in Computer Science and Control (INRIA), Grenoble, France, during 2010-2011. Then, he worked as Research Fellow (academic level 
B) in the Department of Electrical Engineering of the University of Melbourne, Australia, during 20112013. In September 2013, he joined the Department of Electrical Engineering of the Sharif University of
Technology as Assistant Professor. Dr. Farhadi's areas of expertise are networked control systems, distributed optimal control, stochastic and nonlinear controls, and automated irrigation networks. 